\title{
Whither surgical quality assurance of breast cancer surgery (surgical margins and local recurrence) after paterson
}

\author{
N. J. Bundred ${ }^{1}$ J. Thomas' ${ }^{2}$ J. M. J. Dixon ${ }^{3}$
}

Received: 21 June 2017/ Accepted: 29 June 2017/Published online: 5 July 2017

(C) Springer Science+Business Media, LLC 2017

\begin{abstract}
Purpose The Kennedy report into the actions of the disgraced Breast Surgeon, Paterson focussed on issues of informed consent for mastectomy, management of surgical margins and raised concerns about local recurrence rates and the increasing emphasis on cosmesis after mastectomy for breast cancer. This article assesses whether Kennedy's recommendations apply to the UK as a whole and how to address these issues. New GMC advice on consent and newer nonevidenced innovations in immediate reconstruction have altered the level of informed consent required. Patients deserve a better understanding of the issues of oncological versus cosmetic outcomes on which to base their decisions. Involvement of the whole multidisciplinary team including Oncologists is necessary in surgical planning. Failure to obtain clear microscopic margins at mastectomy leads to an increased local recurrence, yet has received little attention in the UK. Whereas, other countries have used surgical quality assurance audits to reduce local recurrence; local recurrence rates are not available and the extent of variation across the UK in margin involvement after surgery, its management and relationship to local recurrence needs auditing prospectively to reduce unnecessary morbidity. To reassure public, patients and the NHS management, an accreditation system
\end{abstract}

N. J. Bundred

nigel.bundred@manchester.ac.uk

1 Department of Surgery, Education and Research Centre, University Hospital of South Manchester, 2nd Floor, Southmoor Road, Manchester M23 9LT, UK

2 Western General Hospital, Edinburgh EH4 2 XU, UK

3 Edinburgh Breast Unit, Western General Hospital, Edinburgh EH4 2XU, UK with more rigour than NHSBSP QA and peer review is now required. Resource and efforts to support its introduction will be necessary from the Royal College of Surgeons and the Association of Breast Surgeons. New innovations require careful evaluation before their backdoor introduction to the NHS. Private Hospitals need to have the same standards imposed.

Keywords Breast Cancer - Local recurrence . Mastectomy $\cdot$ Involved margins

The UK Breast Surgeon, Ian Paterson received a 15 year jail sentence last month after being convicted of intentionally wounding his patients in Private hospitals. He misled vulnerable concerned patients worried about having a breast cancer and persuaded them to undergo unnecessary major surgical procedures rather than reassuring them they had no evidence of cancer.

The Kennedy report [1] into the actions of Paterson in NHS practice focussed on several themes but crucially questioned issues of informed consent, management of surgical margins and raised concerns about local recurrence rates and his emphasis on cosmesis after mastectomy for breast cancer. The report noted a premise widely accepted that "if you cut a cancer out and it is present at the edges (of the cut) there is an increased risk that it will come back at the edge". Kennedy reported that the West Midlands had an overall $1.4 \%$ local recurrence rate following mastectomy, but Paterson had an overall 3\% local recurrence at 5 years, although after Cleavage Sparing Mastectomy (CSM) or following mastectomy and immediate reconstruction, it was $5.7 \%$ after 5 years rising to $9 \%$ by 10 years. Paterson's high rate of local recurrence was explained by Kennedy as due to his nonevidence based 
"CSM" technique which left breast tissue behind and produced a high microscopic margin involvement after mastectomy, despite oncologists mitigating risk with adjuvant radiotherapy [1]. Paterson's prioritisation of cosmesis over complete cancer removal, left margins involved with cancer in 10-37\% of cases undergoing CSM and resulted in a $9-18 \%$ local recurrence rate at 5 years after Mastectomy and Reconstruction [1].

Following local recurrence in the breast there is a 1 in 4 risk of dying from cancer [2]. Internationally, local recurrence rates (LRR) after mastectomy have fallen from $20 \%$ in the 1990 s to $3 \%$ or less, in part due to the increased use of Adjuvant Systemic Therapy. Local recurrence varies with Tumour Phenotype (higher rate in Oestrogen Receptor Negative and HER2 positive cancers), node status and microscopic margin involvement (not type of Mastectomy). The only factor the Surgeon can influence is Margin Status [2, 3].

After Mastectomy for DCIS (when adjuvant therapy is not indicated), Fitzsullivan [4] reported on 810 patients with DCIS, of whom $66 \%$ had Immediate Breast Reconstruction with an overall LRR of $1 \%$ at 5 years. Intraoperative margin assessment by Frozen Section reduced the margin involvement rate from $14 \%$ to a final rate of $7.3 \%$. Margin involvement was associated with a significantly higher 5\% local recurrence rate (which was not affected by post-mastectomy radiotherapy), and was the only independent predictor of local recurrence. A UK study of patients with DCIS found a LRR of $5.9 \%$ in patients undergoing mastectomy and reconstruction with margin involvement a major predictor for LRR. [5] Most of the literature assessing the oncological safety of mastectomy and immediate breast reconstruction comes from single centres with an institutional policy on management of margins. Many series do not report on margin involvement. Reported margin involvement rates in both Europe and USA after mastectomy are low (1-7\%) compared to reported UK rates of up to $29 \%$ [3-7].

In addition to intraoperative frozen section, mastectomy specimen radiology and devices to detect margin involvement [10] are used more widely in the USA but in the UK, there has been a lack of pathology consensus on the need to assess all mastectomy margins. A national quality assurance initiative in Holland that started in 2003, mandated that all Dutch breast surgeons register their mastectomy pathology and for 23,121 patients undergoing mastectomy the rate of involvement of margins fell and correlated with subsequent local recurrence. [7, 8] In 2003, 2.4\% of mastectomies had margin involvement but this reduced to $2 \%$ by 2008. [7, 8] From a previous 3.6\% LRR in 2003 and $30 \%$ of hospitals having a greater than $5 \%$ local recurrences rate, Holland now has a $2.8 \%$ LRR after mastectomy and this compares well to the $2.8 \%$ contralateral breast cancer rate at 5 years. This has led to reduced distant metastasis, reduced cost of both additional chemotherapy and surgery and has improved overall survival [7].

Currently the UK has an extensive audit of many surgical parameters for women diagnosed through the breast screening programme including the number offered immediate reconstruction after mastectomy although no consequences occur for outlying surgeons or units. An audit of UK surgical outcomes in 8313 DCIS patients treated initially by breast conserving surgery looked at reexcision or mastectomy for involved microscopic margins found significant variation in surgical practice between hospitals unrelated to patient factors [9]. There are no UK national audit data looking at variations in the quality of surgery for invasive cancer, margin involvement and local recurrence rates either by hospital or surgeon. Until the 2016 pathology guidelines there was no UK consensus on the evaluation of mastectomy margins and in particular anterior margins after skin-sparing mastectomy. UK data from a series of 570 mastectomy patients in one breast unit found margin involvement that ranged from $15 \%$ after simple mastectomy to $29 \%$ after SSM with a local recurrence rate at 5 years of $5.6 \%$ that increased to $9 \%$ by 8 years in the SSM and reconstruction group. Microscopic margin involvement independently predicted for LRR [3].

The issue of involved margins after mastectomy is rarely discussed with patients, yet the prime concern of most women undergoing mastectomy is complete cancer removal not just cosmesis. Margins of mastectomy specimens need to be considered at multidisciplinary team meetings and when involved it may be appropriate to reexcise margins. When patients are consenting to mastectomy they need to understand that mastectomy may not remove all breast tissue. Re-excision may be required if disease is transected at the mastectomy margin but this should be an infrequent event unlike Paterson's practice. Kennedy makes it clear that mastectomy consent needs to be appropriate, adequate and documented fully.

The UK Association of Breast Surgery (ABS) expectation that LRR should be $<5 \%$ after mastectomy may be too high by current international standards, (particularly for patients with DCIS). LRR after the first 5 years should fall and if it does not then there must be concern that breast tissue may have been left behind [11]. Despite the aim of the $\mathrm{ABS}$ is to reduce the local recurrence rate below $3 \%$ in all breast centres [11], there has been no endeavour to collect data on margin involvement rates or LRR in the UK. Furthermore the UK cannot provide any validated data on cancer outcomes (including LRR and DFS) for individual surgeons or units. The NHS pathology guidelines now require mastectomy margin clearance to be provided in all reports [9, 12]. Data collection (funded in Scotland by the NHS) requires resources and if the rest of the UK is 
serious about collecting information on these outcomes then investment in collecting such data is essential.

The American College of Surgeons requires Breast Units to be accredited and to achieve set standards. Surgeons and Units register their operations to achieve annual accreditation. Outcomes are validated by Independent Surgical monitoring of outcomes arranged by the College. Kennedy recommended rigorous QA screening visits (for Breast Units) that carry sanctions in the event of noncompliance but the data underpinning QA needs to be focussed on oncological outcomes including rates of local recurrence. Is it not now time to follow the US model and have individual units and individual surgeons registering their activity to ensure breast unit accreditation? Individual surgeons fulfilling QA standards for breast cancer outcomes would then be given approval to support appraisal and revalidation. The same standards need to apply in private practice. The time has also come to ensure that surgeons discuss all patients in multidisciplinary teams that include more than a single surgeon both before and after surgery. This will reduce variation in care and ensure that all pathology data are presented and discussed. The NHS improvement initiative group entitled Get it Right First Time (GIRFT) aims to reduce breast practice variation across England, but identifying outcome variation for breast cancer will require a longer term approach. Members of the ABS are involved in this process and need to provide clear and strong leadership to protect patients and reassure the NHS Executive that lessons have been learnt from the Paterson debacle. Importantly, patients must be informed that although mastectomy may not remove all breast tissue, it should remove all invasive and in situ cancer. Mastectomy can be performed safely with or without breast reconstruction though cosmetically placed incisions that are planned as both oncologically safe and aesthetic.

\section{Compliance with ethical standards}

Conflict of interest N.J. Bundred declares that he has no conflict of interest. J Thomas declares that he has no conflict of interest. J.M.J. Dixon declares that he has no conflict of interest.

Ethical approval This article does not contain any studies with human participants or animals performed by any of the authors.

\section{References}

1. Kennedy Report. Review of the response of Heart of England NHS Foundation Trust to concerns about Mr I Paterson's Surgical Practice: lessons to be learned and recommendations

2. Clarke M, Collins R, Darby S, Davies C, Elphinstone P, Evans V, Godwin J, Gray R, Hicks C, James S, MacKinnon E, McGale P, McHugh T, Peto R, Taylor C, Wang Y (2014) Early Breast Cancer Trialists' Collaborative Group (EBCTCG). Effect of radiotherapy after mastectomy and axillary surgery on 10-year recurrence and 20-year breast cancer mortality: metaanalysis of individual patient data for 8135 women in 22 randomised trials. Lancet 383(9935):2127-2135. doi:10.1016/ S0140-6736(14)60488-8

3. Al-Himdani S, Timbrell S, Tan KT, Morris J, Bundred N (2016) Prediction of margin involvement and local recurrence after skinsparing and simple mastectomy. Eur J Surg Oncol 42:935-941

4. Fitzsullivan E, Lari SA, Smith B, Caudle AS, Lucci S, Krishnamurthy A et al (2013) Incidence and consequence of close margins in patients with DCIS treated with mastectomy. Is further therapy warranted? Ann Surg Oncol 20:4103-4112

5. Timbrell S, Al-Himdani S, Shaw O, Tan K, Morris J, Bundred N (2017) Comparison of local recurrence after simple and SSM in patients with DCIS. Ann Surg Oncol 24(4):1071-1107

6. Choong LP, Taib NA, Rampal S, Saad M, Bustam AZ, Yip CH (2010) High tumour stage and margin clearance are still important prognostic factors for post-mastectomy locoregional recurrence in Malaysia. Asian Pac J Cancer Prev 11:1409-1416

7. Aalders KC, van Bommel A, van Dalen T, Sonke G et al (2016) Contemporary risks of local and regional recurrence and contralateral breast cancer in patients treated for primary breast cancer. Eur J Cancer 63:118-126

8. Van der Heiden-van der Loos M, Siesling S, Wouters M, van Dalen $\mathrm{T}$ et al (2012) The value of ipsilateral breast tumor recurrence as a quality indicator: hospital variation in the Netherlands. Breast Cancer Res Treat 131(2):691-698. doi:10. 1007/s10549-011-1809-3

9. Thomas J, Hanby A, Pinder S, Ball G, Lawrence G, Maxwell A et al (2014) Adverse surgical outcomes in screen-detected ductal carcinoma in situ of the breast. Eur J Cancer 50:1880-1889

10. Schnabel F, Boolbol SK, Gittleman M, Karni T, Tafra L, Feldman $S$ et al (2014) A randomized prospective study of lumpectomy margin assessment with use of margin probe in patients with nonpalpable breast malignancies. Ann Surg Oncol 21:1589-1595

11. Association of Breast Surgery at BASO (2009) Surgical guidelines for the management of breast cancer. Eur J Surg Oncol. EJSO. 1:1-22

12. Ellis IO, Al-Sam S, Anderson $\mathrm{N}$ et al. Pathology reporting of breast disease in surgical excision specimens incorporating the dataset for histological reporting of breast cancer. 2016. The Royal College of Pathologists 